\title{
The Force of Habit: Examining the Status Quo Bias for Using Mixed Reality in Patient Education
}

\author{
Eduard Anton \\ Osnabrück University \\ eduard.anton@uos.de
}

\author{
Julian Schuir \\ Osnabrück University \\ julian.schuir@uos.de
}

\author{
Frank Teuteberg \\ Osnabrück University \\ frank.teuteberg@uos.de
}

\begin{abstract}
Using mixed reality (MR) glasses for preoperative patient education (PPE) can help patients understand the purpose and risks of surgical procedures through informative visualizations. However, patients tend to be critical regarding the use of $M R$ glasses in healthcare and often prefer the status quo of healthcare services provided. This study explores the resistance to MR technologies in PPE through the lens of the status quo bias theory by surveying $n=171$ participants. We conducted a fuzzy set qualitative comparative analysis revealing configurations that provide a typological understanding of patient resistance. This allows healthcare stakeholders to take more targeted interventions to promote MR adoption. Notably, the results indicate that healthcare providers need to be transparent in communicating the benefits and drawbacks of using $M R$, as uncertainty costs are the main driver of resistance to MR glasses in PPE.
\end{abstract}

\section{Introduction}

Preoperative patient education (PPE) constitutes a vital component of healthcare services by ensuring patient understanding of upcoming treatments and laying the groundwork for informed consent [1]. It refers to the process of informing individuals about the diagnosis, the treatment, and the associated risks, generally by providing verbal, written, or audiovisual explanations [2]. As such, PPE is one of the most important experiences of healthcare services for influencing patient satisfaction, along with the treatment itself [3]. Despite the importance of PPE procedures, patients often feel inadequately informed about their diagnoses and treatments due to complex terminology, abstract illustrations of procedures, and their lack of health literacy $[4,5]$. A survey of patients undergoing lumbar disc surgery indicates that less than half of the patients are satisfied with their PPE experience [6].

Computer-mediated education applications based on mixed reality (MR) indicate promising potential to resolve these issues by allowing 3D pathology holograms to be visualized and annotated [7, 8]. As Zucker et al. [8:1] state, "these sophisticated visual aids may significantly improve patient understanding of complex anatomy and operative procedures." One example includes Virtual Surgery Intelligence Patient Education (VSI PE), a cloud-based software suite that enables both the physician and patient to experience pictures of magnetic resonance imaging and computed tomography scans in a co-located setting based on the Microsoft HoloLens [7]. In this way, the doctor can demonstrate to the patient the incremental steps of surgery, clarify risks (e.g., injured nerves), and practically illustrate the effectiveness of rehabilitation measures [9]. A recent randomized trial by House et al. [7] revealed that patients perceive PPE through this application to be more imaginable and understandable compared with traditional approaches, such as rubber pathology models, due to the rich visualization capabilities. Despite these advantages, the deployment of wearable MR technologies in professional domains has its drawbacks, such as privacy concerns and physical discomfort [10,11]. For instance, the built-in cameras of MR devices affect bystanders' privacy, and therefore, beta testers wearing the Google Glasses in public were insulted as "glassholes" [12].

To date, however, little is known regarding patients' perception of MR glasses in healthcare [13]. Klinker et al. [14], who examined whether patients would accept healthcare services involving smart glasses, found that they reduce patients' willingness to consent to medical procedures, as the devices increase risk perceptions and decrease patients' assessment of healthcare professionals' skills. Accordingly, patients might be resistant to the idea of using MR glasses in healthcare by preferring to maintain the status quo in line with their habits. Moreover, a crossover trial with elderly patients by Rohrbach et al. [11] revealed that $30 \%$ of the control group tended to be reluctant toward wearing MR glasses due to their novelty and the lack of wearing comfort. In line with these observations, Chuah [15] recently called for illuminating user resistance to MR technologies by employing configurational 
approaches to provide researchers and practitioners with insights into the factors that explain the lack of acceptance of MR glasses.

Responding to this call, the primary objective of this research involves examining patient resistance to MR technologies in the healthcare sector by more closely examining the example of VSI PE. A promising theoretical lens for examining resistance is the status quo bias (SQB) perspective by Samuelson and Zeckhauser [17]. This theory posits factors of rational decision-making, cognitive misperceptions, and psychological commitment that cause resistance to change. To examine the causal combined effect of these resistance-promoting factors, we employ fuzzy set qualitative comparative analysis (fsQCA) [16]. By combining the SQB perspective with the configurational explanatory power of fsQCA, we can uncover new insights into the patient decision-making processes that argue against the use of MR in PPE. Thus, we pose the following research question:

What configurations of conditions induce patients' resistance toward using MR glasses for PPE?

Our findings reveal six configurations that contribute to the research field of MR adoption in healthcare by highlighting the typologies of resistant and non-resistant patients. Healthcare practitioners can leverage these configurations to purposefully select MR devices and applications for PPE and provide individualized information to reduce patient resistance.

The remainder of this paper is as follows: Section 2 describes related work on the adoption of MR technologies in healthcare. Following this, Section 3 presents the research model, after which Section 4 describes the data collection and analysis. The results obtained from the analyses are presented in Section 5, which is followed by a discussion in Section 6. Finally, Section 7 summarizes the findings and discusses the limitations and directions for future research.

\section{Mixed reality in healthcare}

According to Milgram et al. [18], MR is an umbrella term for technologies by which the real world and virtual information are merged, including both augmented reality and augmented virtuality. The present study limits MR to the use of head-worn MR devices, such as MR headsets (e.g., Microsoft HoloLens) that enable users to superimpose 3D holograms into their field of view [19]. To manipulate virtual objects, natural interaction modalities, such as gestures, voice, and facial expressions, are used [20].

Following the advantages of 3D graphics and wearable computing, research has emphasized the use of MR technologies for applications, such as surgery [21] and patient education [7]. In educational settings, the rich visualization capabilities of MR systems facilitate the understanding of complex, otherwise invisible phenomena-for instance, by illustrating the relationships between organs in a tangible fashion [22]. For example, VSI PE provides the ability to explain planned surgery using spatial renderings of computerized tomography (cf. Figure 1).

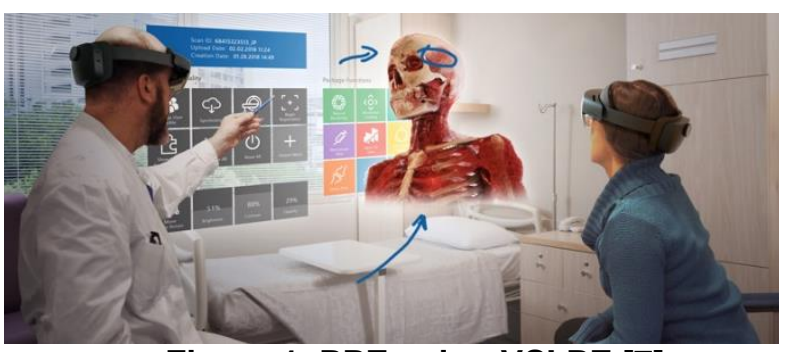

Figure 1. PPE using VSI PE [7]

Growing evidence proves that MR learning fosters long-term retention of learning content, increases motivation, and encourages teamwork [23]. Thus far, however, the vast majority of studies on MR learning focus on the education and training of healthcare professionals (i.e., physicians and nurses), while only a narrow set of studies shed light on the patient aspect of this [13]. Thus, Jakl et al. [23] developed and tested a mobile-based augmented reality prototype to educate strabismus patients about eye surgeries based on interactive 3D storytelling. The authors found that 3D models improve the patient's understanding of surgical procedures and hence should become a complementary tool in PPE. Confirming these findings, House et al. [7] revealed that patients perceive MR-based PPE to be more imaginable and understandable compared with traditional approaches, such as anatomy models.

While the aforementioned studies suggest that 3D visualization itself constitutes a promising avenue for PPE, other studies indicate that the disruptive nature of the hardware and privacy risks lower the acceptance of healthcare services involving MR glasses. For instance, Rohrbach et al. [11] investigated the use of Microsoft Hololens to support daily tasks across Alzheimer's patients. The authors found usability issues, such as wearing comfort, and that the futuristic design of MR headsets might reduce the intention to use them. Moreover, concerns regarding unauthorized access to one's health data and photos generated using the cameras integrated into the devices lower the likelihood of consenting to treatments with MR headsets [14]. This skepticism confirms studies from the consumer sector [15], for instance, by revealing that privacy concerns significantly reduce MR acceptance [24].

However, guidance on how healthcare decisionmakers and MR hardware providers can address the lack of acceptance has remained elusive [19]. Given that 
MR-based PPE is considered a best practice for promoting health literacy [25], this research strives to shed light on the decision-making processes that induce patients' resistance toward using MR glasses for PPE.

\section{Research model}

The focus on resistance differs from conventional adoption research, which typically emphasizes the behaviors that lead to technology acceptance, employing acceptance models such as the theory of planned behavior (TPB), the technology acceptance model (TAM), or the unified theory of acceptance and use of technology (UTAUT) [26]. However, understanding resistance can enable change and initiate measures for enhancing acceptance [27]. We adopt the SQB perspective to explore the resistance of using MRsupported PPE [17]. The SQB perspective argues that it is an oversimplified supposition that patients will select MR-supported PPE over traditional PPE solely because of its perks. Research on decision-making revealed that "[f]aced with new options, decision makers often stick with the status quo alterative" [17:8]. Samuelson and Zeckhauser [17] referred to this deliberate choice in favor of the known custom as SQB. The SQB perspective is used in information systems (IS) research to study decision-making mechanisms that cause resistance when confronted with new IS [26] or with options for setting a new course of action triggered by technology, such as paying with bitcoin [28] or managing one's medication using chatbots [29].

The SQB perspective highlights factors that stem from rational decision-making, cognitive misperceptions, and psychological commitment that condition resistance [26].

We adopt transition costs (TC) and uncertainty costs $(\boldsymbol{U C})$ as trade-offs made at the rational decision level [17]. While TC refer to the time and effort considerations between the alternative and the status quo [28, 29, 30], UC address the information deficit associated with a presented alternative, which requires that patients invest in searching and analyzing information to make an informed decision [29].

We further consider loss aversion (LA), which belongs to the category of cognitive misperceptions and builds on the notion that "individuals weigh losses heavier than gains in making decisions" [17:35]. Previous IS research assessed the presence of LA in an individual when the costs (transition and uncertainty costs) outweigh the perceived benefits $[28,29,30]$. Thus, to measure loss aversion, the perceived benefits $(\boldsymbol{B F})$ of the alternative must be considered.

The next group of factors we consider in our research model pertains to psychological commitment to resist. This includes the decision control $(\boldsymbol{D C})$, which is the perception to what extent the patient has control over the situation and the decision between the alternatives and anticipated regret (AR), which indicates the degree of regrettable consequences, such as safety and education, if the patient would switch to the alternative $[17,26]$.

In addition to these five conditions derived from the SQB perspective, we hypothesize that individual characteristics could additionally influence resistance toward using MR. Acceptance research using the UTAUT often includes individual characteristics as moderators between independent variables and intention to use [31]. Since individual characteristics affect the acceptance mechanisms, we assume that they affect the resistance mechanisms, as well. Therefore, we include gender $(\boldsymbol{G E})$ in light of studies that have demonstrated there are significant differences in computer self-efficacy, in the sense that women tend to have lower perceptions of their technology capabilities $[32,33]$, which might affect cost-related perceptions of effort and time; as such, levels of resistance may differ between women and men.

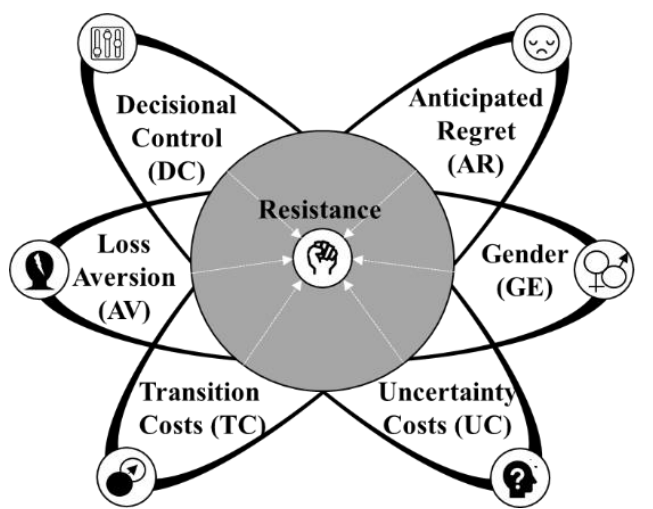

Figure 2. Configurational research model

Considering that resistance is a complex phenomenon, the causality of it typically does not depend on only one factor or the causal symmetry to reasons that lead to acceptance $[28,29]$. Thus, we further enhance our theoretical framework in Figure 2 by building on the complexity and configuration theory to leverage principles of conjunction, asymmetry, and equifinality to address the complex causality [34]. The tenet of conjunction underpins the configurational approach, in which several conditions (state of variable measurements) have a holistic effect on a dependent variable. Asymmetry states that the increase or decrease in the measurement of an independent variable does not necessarily lead to an increase or decrease in the dependent variable (as is the case in symmetric variance-based approaches); rather, the causal conditions leading to the presence or absence of an outcome can differ. Equifinality describes that multiple 
configurations can lead to the same outcome [34]. fsQCA as an approach can capture these principles by providing set-theoretic instruments and Boolean operators to form one or many configurations that lead to the presence or absence of resistance $[28,29]$. In the following section, we present this methodological approach in detail.

\section{Methodological approach}

To address our research objective, we employ an approach that leverages the value of deductive and inductive inquiry $[34,35]$. First, we follow a deductive approach, in which we derive our research model from the SQB theory [17], IS resistance research [26, 28, 29], and literature on MR in healthcare [11]. The derived research model guides the collection of empirical data through an online survey, which we analyze using fsQCA. Our inductive inquiry is based on the elaboration of new ideas and propositions on the foundation of the empirical findings $[34,35]$. The following sections provide additional detail regarding the data collection, measurement model, and fsQCA approach.

\subsection{Data collection}

We conducted an online survey by acquiring workers of Amazon's Mechanical Turk (mTurk). As in previous IS literature $[29,36]$, we consider mTurk to be a useful sampling platform when considering data quality assurance strategies [37], bearing in mind that demand effects can be excluded [38].

Our sampling strategy focused on U.S. citizens to allow for as consistent a cultural sphere as possible to minimize confounding effects. To ensure that the respondents experienced traditional PPE 24 months beforehand, we incorporated unpaid screening questions at the beginning of the survey, following the guidelines of Hunt and Scheetz [37]. We deliberately employed status quo framing [17] at the beginning of the survey, which highlights the traditional PPE of having a physician use rubber models or computerized tomography images to explain the surgical procedure. Furthermore, the introduction outlines MR-supported PPE as a selectable alternative. We described MRsupported PPE based on specifications and images of the tool VSI PE. The introduction was followed by questions that we derived from previously validated constructs, whose items we measured on a 7-point Likert scale. To provide comprehensibility and transparency with the survey, constructs, and measures, we have provided supplementary details at https://bit.ly/3yubIaB. Additionally, we implemented several validity measures throughout the survey to check the workers' attentiveness including a) reverse coded questions and b) manipulation checks, as proposed by Hunt and Scheetz [37].

In total, we surveyed 250 (pre-screened) participants in spring 2021. After data collection, we carefully checked the data regarding the attentiveness by analyzing the completion times, the reverse coded questions as well as the manipulation checks. In this process, we excluded 79 questionnaires, thereby yielding 171 valid responses. The descriptive statistics of the participants are depicted in Table 1 .

Table 1. Descriptive statistics $(n=171)$

\begin{tabular}{|c|c|c|c|c|c|}
\hline Char. & \# & $\%$ & Char. & \# & $\%$ \\
\hline \multicolumn{3}{|c|}{ Age } & \multicolumn{3}{|c|}{ Education level } \\
\hline$<21$ & 1 & $1 \%$ & $\begin{array}{l}\text { High } \\
\text { school }\end{array}$ & 10 & $6 \%$ \\
\hline $21-30$ & 47 & $27 \%$ & $\begin{array}{l}\text { College } \\
\text { degree }\end{array}$ & 161 & $94 \%$ \\
\hline $31-40$ & 57 & $33 \%$ & \multicolumn{3}{|c|}{ Gender } \\
\hline $41-50$ & 35 & $20 \%$ & Male & 74 & $49 \%$ \\
\hline $51-60$ & 20 & $12 \%$ & \multirow{2}{*}{ Female } & \multirow{2}{*}{87} & \multirow{2}{*}{$51 \%$} \\
\hline $60<$ & 11 & $6 \%$ & & & \\
\hline $\begin{array}{l}\text { Legend: } \\
\text { frequen }\end{array}$ & & & & & \\
\hline
\end{tabular}

\subsection{Measurement and validation}

The main information regarding correlations and validity measures is presented in Table 2 (for more detailed information on the calculations of the measurement model, see https://bit.ly/3yubIaB).

Table 2. Measurement and validation

\begin{tabular}{|l|c|c|c|c|c|c|c|}
\hline & $\mathbf{1}$ & $\mathbf{2}$ & $\mathbf{3}$ & $\mathbf{4}$ & $\mathbf{5}$ & $\mathbf{6}$ & $\mathbf{7}$ \\
\hline $\mathbf{1}$ & $\mathbf{. 8 4}$ & & & & & & \\
\hline $\mathbf{2}$ & .05 & $\mathbf{. 7 2}$ & & & & & \\
\hline $\mathbf{3}$ & .00 & .37 & $\mathbf{. 7 5}$ & & & & \\
\hline $\mathbf{4}$ & .30 & .12 & .06 & $\mathbf{. 7 5}$ & & & \\
\hline $\mathbf{5}$ & .35 & .17 & .04 & .60 & $\mathbf{. 8 3}$ & & \\
\hline $\mathbf{6}$ & .24 & .11 & .04 & .66 & .62 &. $\mathbf{7 7}$ & \\
\hline $\mathbf{7}$ & .00 & .01 & .01 & .01 & .01 & .00 & - \\
\hline Legend: Bold diagonal $=$ square root of $\mathrm{AVE} ;$ \\
$1=\mathrm{AR}(\alpha=.80, \mathrm{CR}=.82, \mathrm{AVE}=.70), 2=\mathrm{BF}(\alpha=.85$, \\
$\mathrm{CR}=.85, \mathrm{AVE}=.53), 3=\mathrm{DC}(\alpha=.79, \mathrm{CR}=.79$, \\
AVE $=.56), 4=\mathrm{TC}(\alpha=.74, \mathrm{CR}=.72, \mathrm{AVE}=.57)$, \\
$5=\mathrm{UC}(\alpha=.81, \mathrm{CR}=.82, \mathrm{AVE}=.69)$, \\
$6=\mathrm{RESISTANCE}(\alpha=.86, \mathrm{CR}=.83, \mathrm{AVE}=.59)$, \\
$7=\mathrm{GE}$.
\end{tabular}

The precondition for analyzing latent constructs with the fsQCA approach is the fit of our data to the measurement model; thus, we conducted confirmatory factor analysis [39]. We first performed Harman's single factor test, in which we tested the total variance 
extracted by only a single factor; here, the extracted variance is $34.10 \%$, which is below the threshold of $50 \%$, indicating that there are no major issues with common method bias [40]. Next, we assessed the reliability of internal consistency by calculating both Cronbach's alpha $(\alpha)$ and the composite reliability (CR), which are both greater than .70 for each construct [41]. Furthermore, we ensured construct validity by checking convergent and discriminant validity. For convergent validity, we determined an average variance extracted (AVE) ranging from .53 to .70, which is greater than the critical value of .50 [41]. Moreover, we checked discriminant validity in two ways: first by demonstrating compliance with the Fornell-Larcker criterion, which states that the square root of the AVE of a construct must be higher than the value of the correlations with another factor, and second by calculating the heterotrait-monotrait (HTMT) ratio, which demonstrates that all values are below the upper limit of .85 [42].

\subsection{Fuzzy set qualitative comparative analysis}

Embedded in complexity and configuration theory, fsQCA supports the exploitation of the properties of equifinality, asymmetry, and conjunction [34]. fsQCA is one of several qualitative comparative analysis techniques that is particularly suitable for the social sciences to depict the complex, often ambiguous, phenomena [34]. fsQCA employs what is known as the calibration procedure, in which data is transformed to a value between 0 (indicating full non-membership) and 1 (indicating full membership) based on substantive and empirical knowledge, where .50 constitutes the membership cross-over point. This allows for the representation of intermediate conditions of a measurement, as opposed to the crisp-set alternative (csQCA), which represents the data in absolute terms, (e.g., does the participant express AR [coded as 1] or does he or she not express AR [coded as 0]). We used the direct calibration method [43], employing the QCA package in $\mathrm{R}$. Therefore, we determined three qualitative anchors for full membership, the cross-over point, and full non-membership using the $20^{\text {th }}$ and $80^{\text {th }}$ percentiles as the recommended min-max thresholds (for skewed data) and the median as the cross-over point [39] - except for GE and LA, which were coded binary as a crisp-set. LA was coded as 1 if the mean of the TC and UC exceeded the BF and as 0 if these costs did not exceed the benefits [28]. GE was coded 1 for men and 0 for women.

Based on the calibrated data, set-theoretical analyses were performed to determine which conditions are necessary (supersets of an outcome) and which are sufficient (subsets of an outcome). Conditions within the configurational pool are considered to be necessary if the condition measurements occur with a certain consistency with the outcome (the consistency threshold is typically set to be at least .90 in combination with a coverage threshold greater than .60) [44]. Sufficient conditions are analyzed using the truth-table, which lists all possible configuration combinations that can cause the result $\left(2^{6}\right.$ possible combinations, with six conditions examined to cause resistance). Not all possible combinations can always be observed empirically (socalled limited empirical diversity). In our case, $50 \%$ of the possible 64 combinations could also be empirically confirmed, indicating the validity boundary [45]. Unobserved configurations that are logical remainders are used for further analysis in the Boolean minimization process with the Quine-McCluskey algorithm (counterfactual analysis) [46]. The output of this analysis is a parsimonious, intermediate, and complex solution. We report in the further course the intermediate solution, which includes the parsimonious solution and represents a simplification of the complex solution. Prior to performing the Boolean minimization process, we establish the strength of evidence thresholds, setting the minimum frequency of empirical observations to 3 and a consistency threshold of the recommended .75 , below which all other configurations are treated as logical remainders [39, 45]. Furthermore, we set the threshold for the proportional reduction of inconsistencies (indicates the degree to which a configuration is simultaneously sufficient for the presence and absence of an outcome) to .75 [45]. To further ensure validity in our findings, we performed three robustness tests: a predictive validity test [39], adjusting the calibration thresholds with .90 and .10 as the min-max thresholds [47], and sensitivity tests by varying the strength of the evidence thresholds [45]. The tests confirmed the robustness of the results reported hereafter.

\section{Results}

The set-theoretic analyses in search of necessary and sufficient conditions explaining resistance and $\sim$ resistance (the absence of a condition is notated as " $\sim$ ") were performed using the software fsQCA 3.0. We assessed the relevance of the results by relying on consistency (indicates the significance of the subset relations) and coverage (indicates empirical explanatory power) scores.

Our analysis of necessary conditions revealed only the condition $\sim \mathrm{LA}$, which exceeds the consistency threshold of .90 , as a superset of $\sim$ resistance [48]. This implies that in the absence of LA, the SQB is generally not observed. However, the coverage of .53, which is below the threshold of .60 , indicates that this necessary 
condition has rather low empirical relevance [49]. Since a necessary condition is not sufficient to explain an outcome, we further explored sufficient conditions. For the presentation of these sufficient configurations, we adopt the notation of Ragin and Fiss [50]. Thereby, the presence of a sufficient condition is notated as "๑", while the absence of a condition is indicated by " $\otimes$ ". The size of the icons implies the coreness of a condition within a configuration; as such, small icons are peripheral conditions (based on easy counterfactuals), while large icons are core conditions (based on easy and difficult counterfactuals). Meanwhile, blank spaces represent "don't care"-conditions, the presence or absence of which does not affect the outcome.

Table 3. Sufficient conditions for resistance and $\sim$ resistance

\begin{tabular}{|c|c|c|c|c|c|c|}
\hline & \multicolumn{6}{|c|}{ Configurations } \\
\hline & \multicolumn{3}{|c|}{$\begin{array}{l}\text { Outcome: } \\
\text { resistance }\end{array}$} & \multicolumn{3}{|c|}{$\begin{array}{c}\text { Outcome: } \\
\text { resistance }\end{array}$} \\
\hline & C1 & $\mathbf{C 2}$ & C3 & C4 & $\mathbf{C 5}$ & C6 \\
\hline \multicolumn{7}{|c|}{ Conditions of rational decision making } \\
\hline $\mathrm{TC}$ & $\bullet$ & $\bullet$ & $\bullet$ & $\otimes$ & $\otimes$ & $\otimes$ \\
\hline $\mathrm{UC}$ & 0 & 0 & ○ & $\bigotimes$ & $\bigotimes$ & $\bigotimes$ \\
\hline \multicolumn{7}{|c|}{ Condition of cognitive misperception } \\
\hline LA & & & $\otimes$ & $\otimes$ & $\otimes$ & $\otimes$ \\
\hline \multicolumn{7}{|c|}{ Conditions of psychological commitment } \\
\hline DC & $\bullet$ & & $\otimes$ & & $\otimes$ & \\
\hline AR & • & $\bullet$ & & $\otimes$ & & \\
\hline \multicolumn{7}{|c|}{ Personality trait } \\
\hline $\mathrm{GE}^{*}$ & & $\bullet$ & $\otimes$ & & & - \\
\hline $\mathrm{RCov}$ & .321 & .323 & .136 & .502 & .434 & .297 \\
\hline $\mathrm{UCov}$ & .117 & .158 & .097 & .087 & .064 & .035 \\
\hline RCon & .931 & .940 & .870 & .867 & .865 & .892 \\
\hline SCov & \multicolumn{3}{|c|}{.575} & \multicolumn{3}{|c|}{.632} \\
\hline SCon & \multicolumn{3}{|c|}{.925} & \multicolumn{3}{|c|}{.858} \\
\hline \multicolumn{7}{|c|}{$\begin{array}{l}\text { Legend: } *=\text { presence/negation reflects "male"; } \\
\text { • = presence of an antecedent; } \otimes=\text { negation of an } \\
\text { antecedent; big circle = core element; small circle = } \\
\text { peripheral element; blank space = subordinate } \\
\text { antecedent, RCov = raw coverage, UCov = unique } \\
\text { coverage, RCon = raw consistency, SCon = solution } \\
\text { coverage, SCon = solution consistency. }\end{array}$} \\
\hline
\end{tabular}

SQB is present: The Boolean solution formula that summarizes the configurations leading to the SQB, thus anchoring the known PPE procedure that has been used for decades, is ("**" = logical AND, "+" = logical OR, bold $=$ core condition, not bold $=$ peripheral condition): RESISTANCE $=T C^{*} \boldsymbol{U} \boldsymbol{C}^{*} D C^{*} A R(C 1)+T C^{*} \boldsymbol{U} \boldsymbol{C}^{*} A R^{*}$ $G E(C 2)+T C^{*} \boldsymbol{U} \boldsymbol{C}^{*} \sim L A^{*} \sim D C^{*} \sim G E(C 3)$. The solution formula has a high consistency of .925 and explains more than half of the empirical evidence, resulting in the outcome with a solution coverage of .575. The intersection of all three individual configurations $(\mathrm{C} 1$, $\mathrm{C} 2, \mathrm{C} 3)$ is $\mathbf{T C} * \mathbf{U} \mathbf{C}$, indicating that all decision processes represented by the configurations are rationally guided in particular by the core condition UC. Thus, both the cost-driven perceptions of having to invest time and effort in learning using MR (TC) and the search for information about the innovative alternative (UC) are decisive factors when sticking with the status quo. The differences in the configurations manifest themselves in the peripheral sufficient conditions, which we use to label the configuration profiles.

The committed patient $(\mathrm{Cl})$ is characterized by the fact that this patient profile incorporates conditions from the area of psychological commitment in rational decision-making. Patients represented by this configuration feel that they have control over the decision regarding which option is the best alternative for them, be it the use of MR or the current concept for PPE. Moreover, this configuration is driven by regret, incurring a potentially large loss in terms of education and safety when switching to the MR variant. The regretful male patient $(C 2)$ is characterized by the fact that his cost-driven conclusion to remain in the status quo of the PPE procedure is emphasized by considering regrettable aspects of safety issues and the loss of education in switching. This type is dominated by men and has the greatest empirical relevance of the three configurations. Interestingly, the profile of rational female patients (C3) sticks with the status quo despite the absence of LA, meaning that the BF of the MR alternative prevail for these women. However, this profile does not appear to have control over the decision to selecting between the alternatives.

SQB is absent: The Boolean solution formula representing the configurations leading to the absence of the SQB (i.e., the absence of RE) is $\sim$ RESISTANCE $=\sim T C^{*} \sim U C^{*} \sim L A^{*} \sim A R$ $(C 4)+\sim T C^{*} \sim \boldsymbol{U C} * \sim L A^{*} \sim D C(C 5)+\sim T C^{*} \sim \boldsymbol{U C} * \sim L A^{*}$ $G E$ (C6). The solution formula has a high consistency of .858 and covers a substantial proportion of the empirical cases, resulting in the outcome of $\sim$ resistance with a value of .632. All three resulting configurations are characterized by the same core condition, namely the absence of UC. Therefore, patients overcoming the SQB do not feel any information deficit necessary to compare the alternatives with each other. Furthermore, the intersection of the three individual configurations reveals that for all three, the BF of MR used for PPE exceed the associated burdens ( LA), and they do not perceive much effort in learning how to use MR ( TC). The decision-making process comprising $\sim \mathrm{TC}^{*} \sim \mathrm{UC}^{*} \sim \mathrm{LA}$ can be observed, especially in men (C6, the male alternative seeker). The differences 
between the other configurations are expressed by peripheral conditions:

The remorseless patient $(C 4)$ represents the highest strength of empirical evidence, a profile characterized by the absence of AR in the decision to use MR for PPE. The indecisive patient (C5) feels no $\mathrm{UC}$ or TC and sees the benefits in MR ( LA), but the patient does not feel that he or she has control over selecting one of the options.

\section{Discussion and Implications}

Extant IS research has indicated that the utilization of MR technologies by healthcare professionals negatively impacts consent for treatments and provides limited insight into how patients respond to the use of MR for PPE [14]. Driven by the observation that MR applications, such as VSI PE, offer promising benefits for PPE but are associated with resistance due to their novelty $[11,18]$, this study combines the SQB perspective with configuration and complexity theory to explore the resistance of using MR for PPE. Our results obtained from the fsQCA approach based on a survey of $n=171$ participants who previously experienced the process of preparing for surgery provide insights into the inherent decision-making process that drives resistance to the use of $\mathrm{MR}$ in PPE. In this section, we dive deeper into the identified configurations $\mathrm{C} 1-\mathrm{C} 6$ and its implications, deriving propositions (P) and recommendations $(\mathrm{R})$ for researchers and practitioners.

As research on the adoption of MR technologies has thus far predominantly focused on technology acceptance models, such as the TAM or the UTAUT [15], this study complements prior studies by responding to recent calls by scholars such as Chuah [15] for illuminating resistance toward MR technologies. To the best of our knowledge, this is the first study to shed light on the resistance to MR technologies in the healthcare sector. At the same time, recent research emphasizes the need to capture complex causal phenomena in the socio-technical domain by leveraging conjunction, equifinality, and asymmetry to overcome the shortcomings of variance-based methods $[19,40,60]$. In the context of our research focus, we find three configurations that can explain resistance $(\mathrm{C} 1-\mathrm{C} 3)$ and three configurations that can explain $\sim$ resistance (C4-C6), indicating equifinality where different configurations yield the same outcome. In our case, we observe within-type equifinality, since the configurations are shaped by the same core condition (UC as a core condition for resistance and $\sim \mathrm{UC}$ as a core condition for $\sim$ resistance). Furthermore, we can observe asymmetry because the configurations that explain resistance differ from those that explain the absence of resistance. These findings confirm that a linear perspective on the nature of causality of the SQB is insufficient in capturing the complexity of the underlying decision-making processes. Therefore, we propose the following:

P1: The $S Q B$ in relation to $P P E$ is a complex phenomenon in which the conditions that lead to a $S Q B$ drive the resistance holistically, necessitating a configurational perspective.

Furthermore, we extended the SQB perspective by incorporating the individual characteristic GE as a factor that could influence decision-making. Our results could identify two gender-specific configurations $(\mathrm{C} 2, \mathrm{C} 3)$ for the occurrence of resistance and one configuration for the absence of resistance (C6). Thus far, no attention has been paid in the IS literature to individual characteristics when using the SQB perspective [26, 28, 29]. However, as an integral aspect of the UTAUT model, GE is theorized to have a moderating impact on IS technology acceptance [52], so it should likewise play a role in determining whether it has an impact on RE. Since we have demonstrated that resistance is strongly costoriented in terms of effort, time, and information uncertainty, GE does indeed matter. Social cognitive theory reveals that women have a lower computer selfefficacy and thus a lower assessment of their ability to use technology, which might influence their cost perspective $[32,33]$. Therefore, we argue the following: $P 2$ : To further understand the $S Q B$ with respect to $P P E$, individual characteristics such as GE must be considered to examine its influence on resistance decisions related to the use of $M R$.

We consider the SQB perspective to be insufficient at painting a holistic picture of the configurations that result in resistance to and acceptance of innovative technologies, such as MR. Rather, in line with Kim and Kankahalli [26], we argue it is worthwhile to study the causal relationship between technology acceptance factors (e.g., perceived ease of use) and resistance factors (e.g., TC) [17, 53]. By combining these two perspectives, research can better assess the interplay between acceptance and resistance factors. An example for such a theoretical unification approach is the technology integration model of Shaw et al. [54] which links psychological considerations to technology use models. Such an unification approach may explain the emergence of the SQB, because by accounting for situational context and cost-benefit trade-offs, it can be employed to observe that " $[\mathrm{t}]$ he more a technology is used in response to situational cues rather than as a conscious decision, the more habitual a technology has become" [54:211]. Therefore, we propose the following:

P3: Future research studies on MR adoption should combine acceptance and resistance determinants to 
better understand the interplay between acceptance and resistance factors.

Along with theoretical relevance, our study is expected to provide important implications for practitioners, such as decision-makers in healthcare facilities, physicians, and MR system providers. First, our results reveal that $\mathrm{UC}$ is the core condition for resistance to MR systems in PPE for the configurations $\mathrm{C} 1-\mathrm{C} 3$. This uncertainty suggests that patients struggle to identify the benefits of MR-supported PPE — such as a better understanding of the risks of surgery due to the 3D visualization capabilities [7] - prompting them to reject the technology. To allow healthcare facilities and physicians to counteract this uncertainty prior to PPE, we provide the following recommendation $(\mathrm{R})$ :

R1: Healthcare facilities and physicians should transparently communicate the benefits and drawbacks of using MR in PPE to patients prior to using the technology to lessen patient uncertainty.

Additionally, TC contribute peripherally to resistance in the configurations $\mathrm{C} 1-\mathrm{C} 3$. This finding illustrates that patients perceive the use of MR glasses to be difficult because they are not familiar with the technology and therefore tend to reject it. However, patients actually adopt a passive user role in the use of MR-based applications, such as VSI PE, since the physician can control and manipulate the visualizations as the primary user, allowing the patient to perceive the physician's actions through the MR glasses without actively controlling the device. Thus, MR-based PPE contributes to providing facilitated access to surgicaland disease-related information compared with conventional methods such as verbal descriptions of planned surgery, in which patients often do not understand the planned procedures due to a lack of medical background knowledge [4, 5, 14]. To reduce these perceived $\mathrm{TC}$, we therefore recommend the following:

R2: Healthcare facilities and physicians should stress the ease of use of MR applications for PPE prior to deploying the technology to reduce the perceived transition costs.

Finally, we revealed three configurations that cause resistance toward the use of MR in healthcare due to patients' preference for the status quo, namely the committed patient $(\mathrm{C} 1)$, the regretful male patient $(\mathrm{C} 2)$, and the rational female patients (C3). All three of these configurations share the previously outlined notions of UC and TC but vary with respect to their level of AR, psychological commitment, and sociodemographic characteristics. With this differentiated picture, we offer IT providers and healthcare institutions starting points for the development of configuration-specific communication strategies to reduce the resistance to MR in the PPE [28]. For example, if a patient distinctly expresses a desire to decide for himself or herself which form of patient education he or she would like to use, then he or she belongs to $\mathrm{C} 1$ (i.e., the committed patient). In this case, the physician should presume that the patient expresses AR toward the use of MR and should clearly explain the benefits of the technology. Given this ability to address the concerns of patients, we recommend the following:

R3: IT providers and healthcare facilities should study the configurations produced in the present study to derive configuration-specific communication strategies to effectively reduce patient resistance.

To summarize, this paper provides threefold contributions to the academic literature and healthcare practitioners: First, we contribute to resistance research by uncovering three configurations of factors decisive for the SQB and by identifying three configurations to explain the fact that no SQB occurs. In addition, we provide three propositions to enhance the understanding of future SQB investigations. Second, we contribute to the growing body on MR adoption. To the best of our knowledge, this study is the first attempt at investigating resistance toward MR technologies. Examining resistance in IS can offer insight into the perceived cognitive costs and threats associated with IS and provide starting points for acceptance measures [26]. As stated in P2, future research should combine theoretical acceptance and resistance lenses to enhance the understanding of wearable MR adoption. Finally, we provided three recommendations that can help to lower user resistance toward MR systems in healthcare.

\section{Conclusion, Limitations, and Directions for Future Research}

In this paper, we examined the resistance towards using MR-supported PPE through the lens of the SQB theory. To this end, we conducted a survey with $n=171$ respondents and analyzed the data by conducting an fsQCA. Our findings reveal three configurations that explain resistance and three configurations that lead to $\sim$ resistance towards the use of MR in PPE. In each of the six configurations, it is primarily the degree of UC that determines the presence or absence of resistance. Against this backdrop, we recommend healthcare facilities to transparently communicate the benefits and drawbacks of MR in the PPE to mitigate RE. These findings advance future research on MR in healthcare by outlining a configurational resistance perspective to adoption research.

Despite the findings outlined above, this study is subject to limitations, which in turn can serve as starting points for future research. The first limitation concerns our sampling strategy, which focused on U.S. participants, thereby precluding generalizability to other 
healthcare systems. Nevertheless, we believe that the U.S. healthcare system, which includes the most innovative healthcare providers [55], is suited to experiment with MR offerings in PPE. Nonetheless, future efforts should examine resistance in healthcare systems that are less familiar with innovative healthcare services. Moreover, a challenge pertaining to data collection using the mTurk platform is to ensure data reliability and validity. We followed guidelines from Hunt and Scheetz [37] to ensure that we accessed qualified participants and validated collected data. The second limitation pertains to the conducted fsQCA approach. While it is worthwhile to use fsQCA as the primary method, as is done by many IS researchers [28, 29], the explanatory power can still be increased via complementary quantitative or qualitative analyses [48]. Future studies should incorporate such a complementary approach.

\section{References}

[1] Steves, S.L., and K.N. Scafide, "Multimedia in preoperative patient education for adults undergoing cancer surgery: A systematic review", European Journal of Oncology Nursing 52, 2021, pp. 101981.

[2] Papanastassiou, I., R. Anderson, N. Barber, C. Conover, and A.E. Castellvi, "Effects of preoperative education on spinal surgery patients", SAS Journal 5(4), 2011, pp. 120-124.

[3] Hodgkinson, B., D. Evans, and S. O'Neill, "Knowledge retention from pre-operative patient information", In Database of Abstracts of Reviews of Effects (DARE): Quality-assessed Reviews [Internet]. Centre for Reviews and Dissemination (UK), 2000.

[4] Wittink, H., and J. Oosterhaven, "Patient education and health literacy", Musculoskeletal Science and Practice 38, 2018, pp. 120-127.

[5] Hilt, A.D., K.M. Kapllani, B.P. Hierck, et al., "Perspectives of patients and professionals on information and education after myocardial infarction with insight for mixed reality implementation: Crosssectional interview study", JMIR Human Factors 7(2), 2020.

[6] Rönnberg, K., B. Lind, B. Zoëga, K. Halldin, M. Gellerstedt, and H. Brisby, "Patients' satisfaction with provided care/information and expectations on clinical outcome after lumbar disc herniation surgery", Spine, 2007.

[7] House, P.M., S. Pelzl, S. Furrer, et al., "Use of the mixed reality tool 'VSI Patient Education' for more comprehensible and imaginable patient educations before epilepsy surgery and stereotactic implantation of DBS or stereo-EEG electrodes", Epilepsy Research 159(October 2019), 2020, pp. 106247.

[8] Zucker, B.E., P. Tekkis, and C. Kontovounisios, "Is Reality Limiting Patient Understanding? A Discussion of the Implications of Augmented Reality Technology to Patient Understanding”, Surgical innovation 25(2), 2018, pp. 188-189.
[9] Apoqlar GmbH, "What is Holomedicine?", 2021.

[10] Schuir, J., and F. Teuteberg, "Understanding augmented reality adoption trade-offs in production environments from the perspective of future employees: A choicebased conjoint study", Information Systems and $e$ Business Management, 2021, pp. 1-47.

[11] Rohrbach, N., P. Gulde, A.R. Armstrong, et al., "An augmented reality approach for ADL support in Alzheimer's disease: A crossover trial", Journal of NeuroEngineering and Rehabilitation 16(1), 2019, pp. 111.

[12] Kudina, O., and P.P. Verbeek, "Ethics from Within: Google Glass, the Collingridge Dilemma, and the Mediated Value of Privacy", Science Technology and Human Values 44(2), 2019, pp. 291-314.

[13] Adapa, K., S. Jain, R. Kanwar, et al., "Augmented reality in patient education and health literacy: a scoping review protocol", BMJ open 10(9), 2020, pp. e038416.

[14] Klinker, K., M. Wiesche, and H. Krcmar, "Smart Glasses in Health Care: A Patient Trust Perspective", HICSS, (2020), 3548-3557.

[15] Chuah, S.H.-W., "Wearable XR-technology: literature review, conceptual framework and future research directions", Journal of Technology Marketing 13(3/4), 2019, pp. 205-259.

[16] Ragin, C.C., Redesigning social inquiry: Fuzzy sets and beyond, University of Chicago Press, 2009.

[17] Samuelson, W., and R. Zeckhauser, "Status quo bias in decision making", Journal of risk and uncertainty 1(1), 1988, pp. 7-59.

[18] Milgram, P., H. Takemura, A. Utsumi, and F. Kishino, "Mixed Reality (MR) Reality-Virtuality (RV) Continuum", SPIE Vol. 2351, Telemanipulator and Telepresence Technologies 2351, 1994, pp. 282-292.

[19] Dwivedi, Y.K., E. Ismagilova, D.L. Hughes, et al., "Setting the future of digital and social media marketing research: Perspectives and research propositions", International Journal of Information Management, 2020.

[20] Lee, L.H., and P. Hui, "Interaction Methods for Smart Glasses: A Survey", IEEE Access 6, 2018, pp. 2871228732.

[21] Tang, R., L.-F. Ma, Z.-X. Rong, et al., "Augmented reality technology for preoperative planning and intraoperative navigation during hepatobiliary surgery: a review of current methods", Hepatobiliary \& Pancreatic Diseases International 17(2), 2018, pp. 101-112.

[22] Kamphuis, C., E. Barsom, M. Schijven, and N. Christoph, "Augmented reality in medical education?", Perspectives on medical education 3(4), 2014, pp. 300-311.

[23] Jakl, A., A.M. Lienhart, C. Baumann, et al., "Enlightening Patients with Augmented Reality", Proceedings - 2020 IEEE Conference on Virtual Reality and 3D User Interfaces, VR 2020, 2020, pp. 195-203.

[24] Rauschnabel, P.A., J. He, and Y.K. Ro, "Antecedents to the adoption of augmented reality smart glasses: A closer look at privacy risks", Journal of Business Research 92(April 2016), 2018, pp. 374-384.

[25] Conard, S., "Best practices in digital health literacy", International Journal of Cardiology 292, 2019, pp. 277279.

[26] Kim, and Kankanhalli, "Investigating User Resistance to 
Information Systems Implementation: A Status Quo Bias Perspective", MIS Quarterly 33(3), 2009, pp. 567.

[27] Klaus, T., J.E. Blanton, and S.C. Wingreen, "User Resistance Behaviors and Management Strategies in ITEnabled Change", Journal of Organizational and End User Computing 27(1), 2015, pp. 57-76.

[28] Mattke, J., C. Maier, L. Müller, and T. Weitzel, "Bitcoin resistance behavior: A QCA study explaining why individuals resist bitcoin as a means of payment", ICIS, (2018).

[29] Müller, L., J. Mattke, C. Maier, and T. Weitzel, "Conversational Agents in Healthcare: Using QCA to Explain Patients' Resistance to Chatbots for Medication", In LNCS (including subseries Lecture Notes in Artificial Intelligence and Lecture Notes in Bioinformatics). 2020, 3-18.

[30] Polites, G., and E. Karahanna, "Shackled to the Status Quo: The Inhibiting Effects of Incumbent System Habit, Switching Costs, and Inertia on New System Acceptance", MIS Quarterly 36(1), 2012, pp. 21.

[31] Venkatesh, Morris, Davis, and Davis, "User Acceptance of Information Technology: Toward a Unified View", MIS Quarterly 27(3), 2003, pp. 425.

[32] He, J., and L.A. Freeman, "Understanding the formation of general computer self-efficacy", Communications of the Association for Information Systems 26(1), 2010, pp. 12.

[33] Vekiri, I., and A. Chronaki, "Gender issues in technology use: Perceived social support, computer self-efficacy and value beliefs, and computer use beyond school", Computers \& Education 51(3), 2008, pp. 1392-1404.

[34] Park, Y., P.C. Fiss, and O.A. El Sawy, "Theorizing the Multiplicity of Digital Phenomena: The Ecology of Configurations, Causal Recipes, and Guidelines for Applying QCA", MIS Quarterly 44(4), 2020, pp. 14931520.

[35] Park, Y., O.A. El Sawy, and P.C. Fiss, "The role of business intelligence and communication technologies in organizational agility: A configurational approach", Journal of the Association for Information Systems 18(9), 2017, pp. 648-686.

[36] Mattke, J., C. Maier, L. Reis, and T. Weitzel, "Bitcoin investment: a mixed methods study of investment motivations", European Journal of Information Systems, 2019, pp. 1-25.

[37] Hunt, N.C., and A.M. Scheetz, "Using MTurk to Distribute a Survey or Experiment: Methodological Considerations", Journal of Information Systems 33(1), 2019, pp. 43-65.

[38] Mummolo, J., and E. Peterson, "Demand Effects in Survey Experiments: An Empirical Assessment", American Political Science Review 113(2), 2019, pp. 517-529.

[39] Pappas, I.O., and A.G. Woodside, "Fuzzy-set Qualitative Comparative Analysis (fsQCA): Guidelines for research practice in Information Systems and marketing", International Journal of Information Management 58, 2021, pp. 102310.

[40] Podsakoff, P.M., S.B. MacKenzie, J.-Y. Lee, and N.P. Podsakoff, "Common method biases in behavioral research: A critical review of the literature and recommended remedies.", Journal of Applied Psychology 88(5), 2003, pp. 879-903.

[41] Nunnally, J., and I. Bernstein, Psychometric Theory, McGraw-Hill, New York, 1994.

[42] Henseler, J., C.M. Ringle, and M. Sarstedt, "A new criterion for assessing discriminant validity in variancebased structural equation modeling", Journal of the Academy of Marketing Science 43(1), 2015, pp. 115-135.

[43] Ragin, C.C., "Qualitative Comparative Analysis using Fuzzy Sets (fsQCA)", In Configurational Comparative Methods: Qualitative Comparative Analysis (QCA) and Related Techniques. SAGE Publications, Inc., Thousand Oaks, United States, 2009, 87-122.

[44] Marx, A., and A. Dusa, "Crisp-Set Qualitative Comparative Analysis (csQCA), Contradictions and Consistency Benchmarks for Model Specification", Methodological Innovations Online 6(2), 2011, pp. 103148.

[45] Schneider, C.Q., and C. Wagemann, Set-Theoretic Methods for the Social Sciences, Cambridge University Press, Cambridge, 2012.

[46] McCluskey, E.J., "Minimization of Boolean Functions*", Bell System Technical Journal 35(6), 1956, pp. 14171444.

[47] Maggetti, M., and D. Levi-Faur, "Dealing with Errors in QCA”, Political Research Quarterly 66(1), 2013, pp. 198-204.

[48] Rihoux, B., and C. Ragin, Configurational Comparative Methods: Qualitative Comparative Analysis (QCA) and Related Techniques, SAGE Publications, Inc., 2455 Teller Road, Thousand Oaks California 91320 United States, 2009.

[49] Ragin, C.C., "Set Relations in Social Research: Evaluating Their Consistency and Coverage", Political Analysis 14(3), 2006, pp. 291-310.

[50] Ragin, C.C., and P.C. Fiss, "Net effects analysis versus configurational analysis: An empirical demonstration", In C.C. Ragin, ed., Redesigning social inquiry: Fuzzy sets and beyond. University of Chicago Press, Chicago, 2008, 190-212.

[51] Benbya, H., N. Nan, H. Tanriverdi, and Y. Yoo, "Complexity and information systems research in the emerging digital world", MIS Quarterly 44(1), 2020, pp. $1-17$.

[52] Venkatesh, V., J.Y.L. Thong, and X. Xu, "Consumer acceptance and use of information technology: extending the unified theory of acceptance and use of technology", MIS quarterly 36(1), 2012, pp. 157-178.

[53] Davis, F.D., "Perceived Usefulness, Perceived Ease of Use, and User Acceptance of Information Technology", MIS Quarterly 13(3), 1989, pp. 319.

[54] Shaw, H., D.A. Ellis, and F. V. Ziegler, "The Technology Integration Model (TIM). Predicting the continued use of technology", Computers in Human Behavior 83, 2018, pp. 204-214.

[55] Roy, A., "Introducing the FREOPP World Index of Healthcare Innovation", FREOPP.org, 2020. https://freopp.org/wihi2020-505b1b60bce6 